\title{
HIV-I evolution, drug resistance, and host genetics: The Indian scenario
}

\author{
U Shankarkumar \\ A Pawar \\ K Ghosh \\ National Institute \\ of Immunohaematology (ICMR), KEM \\ Hospital, Parel, Mumbai, Maharashtra, \\ India
}

\begin{abstract}
A regimen with varied side effects and compliance is of paramount importance to prevent viral drug resistance. Most of the drug-resistance studies, as well as interpretation algorithms, are based on sequence data from HIV-1 subtype B viruses. Increased resistance to antiretroviral drugs leads to poor prognosis by restricting treatment options. Due to suboptimal adherence to antiretroviral therapy there is an emergence of drug-resistant HIV-1 strains. The other factors responsible for this viral evolution are antiretroviral drug types and host genetics, especially major histocompatibility complex (MHC). Both primary and secondary drug resistances occur due to mutations in specific epitopes of viral protein regions which may influence the $\mathrm{T}$ cell recognition by immune system through MHC Class I and class II alleles. Mutations in viral epitopes enable the virus to escape the immune system. New drugs under clinical trials are being added but their exorbitant costs limit their access in developing countries. Thus the environmental consequences and, the impact of both viral and host genetic variations on the therapy in persons infected with HIV-1 clade C from India need to be determined.
\end{abstract}

Keywords: HIV-1 C drug resistance, virus adaptation, HARRT, India

\section{Introduction}

India had an estimated 2.4 million HIV-infected individuals at the end of 2008. ${ }^{1}$ The introduction of cost-effective generic antiretrovirals (ARVs) since 2000 has shown ARVs to be a safe, tolerable, efficacious, and effective treatment modality, ${ }^{2}$ thereby decreasing the morbidity and mortality due to HIV/AIDS. ${ }^{3}$ ARV drug classes include entry and fusion inhibitors, nucleoside reverse transcriptase inhibitors, nonnucleoside reverse transcriptase inhibitors, integrase inhibitors, and protease inhibitors. At present, 30 drugs are available for three different combination regimens. The approach is known as highly active antiretroviral therapy (HAART). In India, the National AIDS Control Organization (NACO) recommends HAART to all AIDS patients. However, these benefits are overshadowed to a greater extent by the drug-resistant forms of the virus. Most of the drug resistance studies, as well as interpretation algorithms, are based on sequence data of HIV-1 subtype B viruses. Non-B subtypes are predominant in the developing countries like India and extensive studies of resistance mutations in treated and untreated patients may help in updating the existing algorithms.

Recent studies indicate subtype $\mathrm{C}$ is responsible for majority of the infections worldwide $(>56 \%)$ and $>98 \%$ of the infections in the Indian subcontinent. ${ }^{4}$ In addition, recombinants $\mathrm{A} / \mathrm{C}$ and $\mathrm{B} / \mathrm{C}$ have also been reported. ${ }^{5,6} \mathrm{~A}$ number of in vitro and in vivo observational studies suggest that the currently available protease and reverse transcriptase (RT) inhibitors are as active against non-B viruses as against subtype B viruses. ${ }^{7}$ However, a few studies also suggest that various subtypes may respond differently to some antiretroviral drugs. ${ }^{8,9}$ The frequency of one or more major resistance mutations in recently infected individuals increased from 3.8\% to $10.2 \%$ under treatment in a five-year period as reported by the West. ${ }^{10}$ Despite widespread use of ARV agents and increasing 
incidence of drug-resistant forms of HIV among both untreated (primary resistant) and treated (secondary resistant) individuals worldwide, only sparse information is available on the prevalence of HIV-1 drug resistance from India.

\section{Primary drug resistance}

Current studies on transmission of drug resistance consider three classes of antiretroviral drugs (nucleoside reverse transcriptase inhibitors [NRTIs], non-NRTIs [NNRTIs], and protease inhibitors). But since 2003, the fusion inhibitor enfuvirtide is also available in clinical practice. Transmitted resistance to enfuvirtide has been documented. ${ }^{11}$ In recent years, antiretroviral treatment in India has been expanded, however studies on drug resistance are quite rare. Few earlier studies on drug resistance in antiretroviral therapy (ART)naïve Indian patients carried out in southern part and south western part of India revealed no primary NRTI and NNRTI drug resistance mutations. ${ }^{12,13}$ However $1.7 \%$ prevalence of primary drug resistance was reported from Mumbai. ${ }^{14,15}$ A similar study from Chandigarh, in the northern part of India, reported a high prevalence of M184 V/I (32\%) and K70R (78.3\%) and a low prevalence of T215 Y/F (1.67\%) among ART-naïve patients. ${ }^{16}$

Apart from host genetic changes, extensive viral genetic variations (minor mutations) in pol gene were seen in Indian HIV strains; most of them being polymorphic specific to subtype C. In the NRTI region the amino acid substitutions at positions 13, 20, 22, 35, 39, 48, 60, 67, 118,177, 200, 207, 214, 245 , and 210 may be associated with drug resistance whereas, in the NNRTI viral region substitutions are at positions 103, 230, and 238. ${ }^{11}$ An exceptional study to determine the prevalence of primary resistance in 460 viral isolates of 74 individuals from Mumbai revealed overall resistance against RT inhibitors as 33.7\% while among protease inhibitors including saquinavir as $21.5 \% .{ }^{15}$ Polymorphisms at M36, L63, and I93 were observed in more than 90\% of the sequences when compared to earlier studies. ${ }^{11-16}$ Recently $10 \%$ of primary resistance mutation V82A in protease gene and; M41L, D67N, M184V, and A98G in RT gene were observed in HIV-1-infected ART-naïve patients from western India. ${ }^{17}$ From north India M46I was identified in protease region of the pol gene and G190V of RT gene. ${ }^{18}$ However, no resistance mutation was identified in protease gene of nine patients studied from south India. ${ }^{19}$

\section{Secondary drug resistance}

Earlier studies on HIV-1 clade C have shown that a V106M mutation confers cross-resistance to NNRT inhibitor ${ }^{20}$ and further they develop K65R resistance to tenofovir in cell culture. ${ }^{21}$ A recent study from Chennai as the most common among NRTI mutations, followed by mutations at codons 44 (15.6\%), 69 (13.7\%) and 74 (6.4\%) whereas K65R and Q151M were observed in only $<2 \%$. Among the NNRTI mutations K103N, G190A, and Y181C were predominant (26\%-28\%). ${ }^{22}$ In a study from Pune, at least one HIV drug-resistant mutation in RT was observed in $80.55 \%$ of ARV-experienced patients and additionally, M184V was the major mutation (69.7\%) seen in the study participants culminating in treatment failure, but K65R mutation was observed. ${ }^{23}$ Others reported NNRTI drug-resistant mutations among those who received single dose nevirapine (SD-NVP) at $48 \mathrm{~h}$ (10.5\%) and at two months post-partum (46.2\%), but left the commonest K103N mutation unseen. ${ }^{24}$

In addition, a very high prevalence of drug resistance to NRTI and NNRTI ( $>90 \%$ ) has been recently observed in Chennai. ${ }^{19}$ The first report on proteinase (PR) resistance among treatment-failing patients in India has been reported, where no primary PR drug resistance mutations were detected. ${ }^{22-25}$ Compared to the number of persons living with HIV in India, a very small number of them have been assessed and reported with variables of interest. Therefore more research on ARV resistance from India is warranted. In summary, transmission of resistance has been reported in all parts of the world. The size of the problem varies between $0 \%$ and $25 \%$ and seems to be the highest in areas where ARVs have been available for a long period of time. ARVs have shown to dramatically decrease morbidity and mortality among people living with HIV and are therefore increasingly provided in many parts of world. Monitoring of transmitted resistance continues to be needed to allow a timely modification of ART guidelines.

\section{HLA-associated mutations}

The antigen presentation, $\mathrm{T}$ cell activation and $\mathrm{T}$ cell receptor binding are complex processes. Antiviral cytotoxic T lymphocytes (CTLs) kill HIV-infected cell upon by recognition through specific viral epitopes "Hot spot" wherein epitopes cluster in immunodomimant regions of HIV protein. ${ }^{26}$ The viral escape mutations at critical binding sites interfere with proteosomal antigen processing of viral antigens by proteasome human leukocyte antigen (HLA)restricted killing. ${ }^{26-28}$ Thus HIV escapes antiviral immune responses and clearance by the host immune system. Such selection pressure as well viral adaptation to antiretroviral drugs may lead to viral quasispecies with different amino acid sequences. Though HLA A and B present viral epitopes to 
CD8 T cells causing cell destruction, HIV-1 accessory protein Nef downregulates their expression on the surface of infected cells. A number of associations of major histocompatibility complex (MHC) class I-dependant CTL responses shape the viral sequence by directly killing infected cells that present the right epitope in their HLA molecule are targeted by CTLs and continuous pressure by CTL responses leads to the selection of survival dominant HIV-1 strains that have mutations in these regions. The continuing selection pressure by $\mathrm{T}$ cell recognition prevents the virus from reverting back despite changes in the antiviral drug regimen. In Western Australia, MHC class I alleles have been shown to render the virus to evolve at population level. ${ }^{27-34}$ Moore and colleagues ${ }^{34}$ have shown 64 positive and 17 negative associations of HLA A or HLA B with viral mutations mainly located in known CTL epitopes. Certain regions in RT and protease are targeted by a greater number of epitopes and therefore CTLs, thus driving viral evolution in this region. The HLA-driven evolution of a fragment of the RT as well as protease of the HIV-1 virus has been previously reported. Further interactions of antiretroviral drugs and HLA alleles with diverse viral RT and protease viral sequences led to have differential frequencies of HLA alleles. ${ }^{29}$ Ahlenstiel and colleagues ${ }^{35}$ have identified 12 significant HLA associated mutations in $\mathrm{RT}$, four lying outside the $\mathrm{RT}$ region: $\mathrm{HLAB} * 51$ at amino acid position 135 , B $* 07$ at position 162 , $\mathrm{A} * 11$ at position 166 , and $\mathrm{B} * 35$ at position 177 . Also, out of 11 new associations, six mutations in the RT sequence are located in known epitope anchor positions. ${ }^{35}$

Unlike expression of HLA-A and B, HLA C expression is unaffected so as to restrict HIV-1 specific genetic associations. So far, the impact of MHC class II alleles on viral evolution has been studied only for a small patient group. Harcourt and colleagues $^{36}$ described HIV sequence variation in the p24 Gag epitope of HLA-DR1. A role of MHC Class II alleles and CD4 T cell responses in the evolution of the HIV-1 sequence is obvious by two associations in the RT region. Interestingly, six of the identified associations were negative associations, indicating that mutations in the RT were less likely if the patient carried that particular allele: amino acid position 177 was negatively associated with HLA-B $* 35$, position 178 with HLA-B $* 35$, position 188 with HLA-DRB $1 * 12$, position 207 with $\mathrm{B} * 15$, position 277 with HLA-A $* 03$, and position 291 with HLA-B $* 27$, HLA-DRB1-associated mutations in the RT (position 67 for HLA-DRB1 * 08 and position 188 for HLA-DRB1 * 12). ${ }^{35}$ Studies have reported that the relative contribution of HLA-B alleles outweighs the contribution of HLA-A alleles and DRB1 alleles with HLA-B alleles $(68 \%)$ than with HLA-A alleles $(23 \%)$ or HLA-DRB1 alleles $(9 \%) .{ }^{37}$ This indicates that HLA-dependent specific immune responses can support but also prevent the evolution of drug resistance.

\section{Future perspectives}

When compared to the alert cutoff (5\%), the prevalence of known drug-resistance in treatment naive HIV-1 patients is very low. In depth studies on the environmental consequences and, the impact of both viral and host genetic variations are necessary on the HIV-1 clade C-infected persons. Availability of affordable plasma viral load testing for drug monitoring and viral and host genotyping would help in HAART efficacy for better clinical management of the disease.

\section{Disclosure}

The authors report no conflicts of interest in this work.

\section{References}

1. AIDS epidemic update. Oct, 2008. [Cited on Jan 10, 2009]. Available from: http://www.who.int/globalatlas/predefinedReports/EFS2008/full/ EFS2008_IN.pdf.

2. Kumarasamy N, Solomon S, Chaguturu SK, et al. The safety, tolerability and effectiveness of generic antiretroviral drug regimens for HIV- infected patients in South India. AIDS. 2003;17:2267-2269.

3. Palella FJ Jr, Delaney KM, Moorman AC, et al. Declining morbidality among patients with advanced human immunodeficiency virus infection, HIV out patient study Investigators. NEng J Med. 1998;338: 853-860.

4. Robertson DL, Anderson JP, Bradac JA, et al. HIV-1 Nomenclature proposal. Science. 2000;288:55-56.

5. Lole KS, Bollinger RC, Paranjapae RS, et al. Full-length human immunodeficiency virus type 1 genomes from subtype $\mathrm{C}$ infected seroconverters in India, with evidence of intersubtype recombination. J Virol. 1999;73:152-160.

6. Tripathy SP, Kulkarnai SS, Jadhav SD, et al. Subtype B and subtype C HIV type 1 recombination in the northeastern state of Manipur India. AIDS Res Hum Retroviruses. 2005;21:152-157.

7. Weidle PJ, Malamba S, Mwebaze R, et al. Assesment of a pilot antiretroviral drug therapy programme in Uganda patients' response survival an drug resistance. Lancet. 2002;360:34-40.

8. Ariyoshi K, Matsuda M, Miura H, Tateishi S, Yamada K, Sugura W. Pattenrns of point mutations associated with antiretroviral drug treatment failure in CRF01 Ae (subtype E) infection differ ffrom subtype B infection. J Acquir Immune Defic Syndr. 2003;33:336-342.

9. Brenner B, Routy JP, Quan Y, et al. Persistance of multidrug resistance HIV-1 in primary infection leading to super infection. AIDS. 2004;18:1653-1660.

10. Little SJ, Holte S, Routy JP, et al. Antiretroviral drug resistance among patients recently infected with HIV. N Eng J Med. 2002;347:385-394.

11. Van de Vijver D, Wensing A, Boucher C. The epidemiology of transmission of drug resistant HIV-1. In: Leitner T, Foley B, Hahn B, et al, editors. HIV Sequence Compendium 2006/2007. Los Alamos, NM: Theoretical Biology and Biophysics Group, Los Alamos National Laboratory, LA-UR 07-4826;2007.

12. Balakrishnan P, Kumarasamy N, Kantor P, et al. HIV type 1 genotypic variation in an antiretroviral treatment-naïve population in Southern India. AIDS Res Hum Retroviruses. 2005;21:301-305.

13. Soundararajan L, Karunajanandham R, Jauvin V, et al. Characterization of HIV-1 isolates from antiretroviral drug naïve children in southern India. AIDS Res Hum Retroviruses. 2007;23:1119-1126. 
14. Eshleman SH, Hudelson SE, Gupta A, et al. Limited evolution in the HIV type 1 pol region among acute seroconverters in Pune. India. AIDS Res Hum Retroviruses. 2005;21:93-97.

15. Deshpande A, Recordon-Pinson P, Deshmukh R, et al. Molecular characterization of HIV type 1 idsolates from unrelated patients of Mumbai (Bombay) India an detection of rare resistance mutations. AIDS Res Hum Retroviruses. 2004;20:1032-1035.

16. Hira SK, Panchal K, Parmar PA, Bhatia VP. High resistance to antiretroviral drugs the Indian experience. Int J STD AIDS. 2004;15:173-177.

17. Sachdeva N, Sehgal S, Arora SK. Frequency of drug resistant variants of HIV-1 coexistent with wild-type in treatment naïve Patients of India. Med Gen Med. 2005;7:68

18. Lall M, Gupta RM, Sen S, Kaplia K, Tripathi SP, Paranjape RS. Profile of primary resistance in HIV-1 infected treatment-naïve individuals from western India. AIDS Res Hum Retroviruses. 2008;24:987-990.

19. Arora SK, Gupta S, Toor JS, Singalia A. Drug resistance-associated genotypic alterations in the pol gene of HIV type 1 isolates in ARTnaïve individuals in North India. AIDS Res Hum Retroviruses. 2008;24: 125-130.

20. Brenner B, Turner D, Oliveira M, et al. A V106M mutation in HIV-1 clade $\mathrm{C}$ viruses exposed to efavirenz confers cross- resistance to non-nucleoside reverse transcriptase inhibitors. AIDS. 2003;17:F1-F5.

21. Brenner B, Oliveira M, Doualla-Bell F, et al. HIV-1 subtype C viruses rapidly develop $\mathrm{K} 65 \mathrm{R}$ resistance to tenofovir in cell culture. AIDS. 2006;20:F9-F13.

22. Kandathil AJ, Kannangai R, Abraham OC, SudharsanamTD, Pullimood SA, Sridharan G. Genotypic resistance profile of HIV-1 protease gene: a preliminary report from Vellore, South India. Ind $J$ Med Microbiol. 2008;26:151-154.

23. Sen S, Tripathy SP, Patil AA, Chimanpure VM, Paranjapae RS. High prevalence of human immunodeficiency virus type 1 drug resistance mutations in antiretroviral treatment experienced patients from Pune. India. AIDS Res Hum Retroviruses. 2007;23:489-497.

24. Deshpande A, Jauvin V, Magnin N, et al. Resistance mutations in subtype C HIV type 1 isolates from Indian patients of Mumbai receiving NRTIs plus NNRTIs and experiencing a treatment failure: resistance to AR. AIDS Res Hum Retroviruses. 2007;23:335-340.

25. Kurle SN, Gangakhedkar RR, Sen S, Hayatnagarkar SS, Tripathy SP, Paranjapae RS. Emergence of NNRTI drug resistance mutations after single-dose nevireprine exposure in HIV type 1 subtype co-infected infants in India. AIDS Res Hum Retroviruses. 2007;23:682-685.
26. Brown SA, Lockey TD, Slaughter C, et al. T cell epitope "hotspots" on the HIV type 1 gp120 envelope protein overlap with tryptic fragments displayed by mass spectrometry. AIDS Res Hum Retroviruses. 2005;21:165-170.

27. Korber, BTM, Brander C, Haynes BF, et al, editors. HIV Molecular Immunology 2005. Los Alamos, NM: Los Alamos National Laboratory, Theoretical Biology and Biophysics. 2005.

28. Allen TM, Altfeld M, Yu MXG, et al. Selection, transmission, and reversion of an antigen-processing cytotoxic T-lymphocyte escape mutation in human immunodeficiency virus type 1 infection. J Virol. 2004;78:7069-7078.

29. Yokomaku Y, Miura H, Tomiyama H, et al. Impaired processing and presentation of cytotoxic-T-lymphocyte (CTL) epitopes are major escape mechanisms from CTL immune pressure in human immunodeficiency virus type 1 infection. J Virol. 2004;78:1324-1332.

30. Zimbwa P, Milicic A, Frater J, et al. Precise identification of a human immunodeficiency virus type 1 antigen processing mutant. $J$ Virol. 2007;81:2031-2038.

31. Draenert R, Le Gall S, Pfafferott KJ, et al. Immune selection for altered antigen processing leads to cytotoxic T lymphocyte escape in chronic HIV-1 infection. J Exp Med. 2004;199:905-915.

32. McMichael AJ, Rowland-Jones SL. Cellular immune responses to HIV. Nature. 2001;410:980-987.

33. John M, Moore CB, James IR, Mallal SA. Interactive selective pressures of HLA-restricted immune responses and antiretroviral drugs on HIV-1. Antivir Ther 2005;10:551-555.

34. Moore CB, John M, James IR, Christiansen FT, Witt CS, Mallal SA. Evidence of HIV-1 adaptation to HLA-restricted immune responses at a population level. Science. 2002;296:1439-1443.

35. Ahlenstiel G, Kristen R, Martin D, et al. Selective pressures of HLA genotypes and antiretroviral therapy on human immunodeficiency virus type 1 sequence mutation at a population level. Clin Vaccine Immunol. 2007; 14:1266-1273.

36. Harcourt GC, Garrard S, Davenport MP, Edwards A, Phillips RE. HIV-1 variation diminishes CD4 T lymphocyte recognition. J Exp Med. 1998;188:1785-1793.

37. Kiepiela P, Leslie AJ, Honeyborne I, et al. Dominant influence of HLA-B in mediating the potential co-evolution of HIV and HLA. Nature. 2004;432:769-775. 